\title{
Modelling the Indigenous virtual self using fuzzy logic as a data filtering system
}

\author{
Kevin Shedlock' ${ }^{(i D}$ and Marta Vos ${ }^{2}$
}

\begin{abstract}
Virtual reality offers tremendous opportunities for Indigenous communities to experience themselves inside unreal settings not available in the real world. However, virtual reality as a technology is complex involving both an internal and external connection that is usually dependent on one's attention shifting from the physical Indigenous environment to the virtual reality environment. Any deviance from heritage or known Indigenous relationships may result in a disconnected drift between the traditional and unreal digital world if wrongly presented. The concern virtual reality maybe replacing the traditional identity of users with a digital version of themselves. At the centre of this problem is the technology itself, not being exposed to Indigenous concepts during the construction of any virtual reality IT artefact? This study applies a multidisciplinary approach to tackle a key issue for virtual reality by locating a version of Indigenous identity of self within a digital setting during the construction of the virtual reality artefact.
\end{abstract}

\section{Keywords}

Indigenous IT artefact, Indigenous digital self, Indigenous science, virtual reality

\section{Introduction}

In many cases, practical problems can be solved by means of artefacts. An artefact is described as an object made by humans with the intention that it be used to address a practical problem (Shedlock \& Vos, 2019). Some artefacts are physical objects while others take the form of drawings or blueprints, such as an architect's plan used for building homes. For Indigenous communities, artefacts can be carvings, flax baskets, weapons or symbols etched into surfaces.

As the world enters into the 4th Industrial Revolution Hartwell (2017) the global community stands on the cusp of exponential change in search of a technology merge of the physical, digital and biological ecosystems Suh et al. (2014) altering the world we live-in (Shedlock et al., 2016). A number of industries will advance in technology disciplines such as robotics, the internet of things, autonomous vehicles and virtual reality (VR) environments alike. Indigenous people' are starting to embrace VR revisiting un-told stories, attending virtual meetings and, finding new ways to enact with virtual traditions. However, the danger is the IT artefact construction process itself is not considering Indigenous practices, processes or concepts during its construction. This may be leading to a possible spatial disconnection experience for Indigenous VR participants during and post construction of the IT artefact. The challenge of replacing the Indigenous traditional self with a virtual representation becoming a profound challenge to tradition, heritage and self-identity posing a growing concern.

This study shows how a discretely organized model of theory can be implemented to address a key challenge for
Indigenous VR in research. Stated further by Shedlock and Vos (2019) as a need to build foundation theory using Indigenous concepts, ideas and understanding during the process of construction knowledge:

"It is difficult if not impossible to make much progress in the application without theory; conversely, it is difficult to understand the theory without knowledge of the technique" as cited in (Gregor \& Hevner, 2013).

While Indigenous research and VR present a multidisciplinary approach with two large domain topics available to enquiry, we keep the scope of the investigation limited to an Indigenous post-colonial approach of enquiry. One that applies a lens to locating a VR presence using a sense of self-agency during the modelling phase of the IT artefact construction.

\section{Problem statement}

As a portal, VR offers a sense of being located in an Indigenous world allowing users to re-visit untold stories, meet in unreal spaces and experience traditional practices. However, VR as a technology presents a danger to the way Indigenous peoples communicate, and the way Indigenous people define their digital selves (Shedlock et al., 2016).

IVictoria University of Wellington, New Zealand ${ }^{2}$ Whitireia Polytechnic, New Zealand

\section{Corresponding author:}

Kevin Shedlock, Victoria University of Wellington, Kelburn, Wellington 6012, New Zealand.

Email: Kevin.shedlock@vuw.ac.nze 
Extending the risk of disconnecting Indigenous users from their known traditional selves.

For Indigenous researchers to respond and benefit from new and emerging technology, Indigenous researchers must continue to seek their own understanding and awareness of how technology such as VR exists. Thereafter, report those findings as Indigenous research activities. This leads to the research question: can VR image-processing techniques be modelled to connect Indigenous participant users to their digital selves?

To respond, first we position the study as an Indigenous science system of theory using Indigenous research. Second, the study reveals the importance of VR as a technology to establish a link to Indigenous stories. Finally, we review evolutionary computing in the form of a FLS to create an Indigenous data filtering method for organizing an Indigenous assessment of self using Indigenous objects, terminology's and languages during the construction of the VR artefact.

\section{Method}

The method for this study follows Design Science Research from Gregor and Hevner (2013) involving seven steps. (1) Beginning with a problem statement introduced early in the research cycle. (2) The literature uses prior relevant work and theories from Indigenous content experts as a channel for framing an Indigenous mode of research during the production phase of Indigenous knowledge. Where Indigenous research does not exist or lacks detail, eurocentric research paradigms are discussed and framed to reflect Indigenous notions and ideas. (3) The artefact exists in the form of a process-model designed as an architectural blueprint and framed as being Indigenous. (4) Evaluation involves the literature and the IT artefact mapping the design process and the design product (meta-design) to achieve the meta-requirements of evaluation (Pries-Heje et al., 2008). Evaluation anchors two key themes: the evaluation of design and, the evaluation of the construct prior to construction, which involves how, what, when and where evaluation occurs during the research activity. (5) Discussion provides an overview of the research topic and seeks to aid both the research environment and, the related body of knowledge's kernel theories. (6) The conclusion completes the research cycle with findings stated as new contributions to the research topic. Hevner and Chatterjee (2010) prescribe two key contributions for any research effort to be successful. The first being the relevance of research that targets the research environment i.e., research connecting the Indigenous community to knowledge through VR. The second being, the rigour applied to the domain knowledge base under examination in search of a contribution to existing kernel theory (Hevner and Chatterjee, 2010).

\section{Literature review}

To address the research question, the literature considers a post-colonial Indigenous approach towards research then, introduces prior Indigenous research to position the study. Essential to keeping the study focused towards Indigenous paradigms, methods and artefacts in support of Indigenous knowledge gathering activities leading the construction of the VR artefact. Thereafter, VR is explored as a technology connecting the Indigenous virtual-self. Finally, the literature presents evolutionary computing as a fuzzy data filter method in support of an algorithm using VR.

\section{Indigenous post-colonial approach towards technology}

Researchers continue to work on decolonizing methods to address challenges in the digital era framing theoretical foundations of postcolonial Indigenous research from early decolonizing methodologies from the likes of (Agrawal, 1995; Battiste et al., 2002; Dei, 2000; Smith, 1999; Wilson, 2001). Based on the assumption that grievance settlement processes are occurring, post-colonial efforts in Indigenous research become a distinct possibility. Stated further,

We have to learn to think anew - to think in ways that seriously and actually respond to information, understanding and knowledge as if difference confronts us with the possibility of thinking differently. (Johnson et al., 2016, p. 3)

In a ubiquitous era, researchers have sought to position an Indigenous research paradigm as a movement of decolonization with the aim to empower and provide a central Indigenous way of knowing and being through culturally appropriate research processes and protocols (Pidgeon, 2019). In this way, decolonizing methodologies provide guidance for transformational change. A post-colonial search scaled to interact with cosmological relationships and processors that overlap with human and non-human presence and responsibilities (Hsu et al., 2014). Some research show concerns towards postcolonial Indigenous research citing "the recovering, valuing, and internationalizing of postcolonial Indigenous epistemologies, methodologies, and methods as a pending problem" (Chilisa, 2012, p. xvi). Others state postcolonial Indigenous paradigms bear a close resemblance to eurocentric research where holistic connections to the colonized can learn to analyse and understand their circumstance to change (Freire, 2000).

In response, an Indigenous technology paradigm must move beyond a eurocentric approach by creating Indigenous technological models, frameworks and systems when researching the sciences (Shedlock \& Vos, 2018, 2019). Indigenous researchers must continue to pursue technology concepts moving from individual knowledge to one that is relationally bound as a shared collective knowledge and continue to progress from the earlier works of Indigenous researchers (Grant et al., 2010; Henry \& Pene, 2001; Smith, 1999; Tuhiwai \& Reid, 2000; von Thater-Braan, 2007; Wilson, 2003).

\section{Indigenous knowledge of the artefact sciences}

Indigenous people are proactive creators of the artificial embracing new technologies such as creating tools for 
ocean navigation, preserving food, weapons for hunting and warfare, making clothing, and building dwellings as well as recording knowledge using known events depicted in carvings, weaving, tattooing, storytelling and music (Bennett, 2012; Bird, 2018; Boxall et al., 2003; A. Harris, 2014). The Indigenously constructed artefact establishes a connection to an event or thing that may have occurred tens of thousands of years before, with the resulting knowledge passed down through the generations. There is reasoning attached to the artefact creation strengthening the Indigenous community's practices in respect of their language, terminology, symbols, patterns and political processing as part of local community systems (Hennessy $\&$ Nathan, 2014). The artefact is considered to be a living treasure crafted through the skill-full knowledge of expert creators forming a structure permeated with the breadth of life (von Thater-Braan, 2007).

These creators are provided with status as knowledge holders and ancestral communicators of technology such as:

- Aboriginal Australia - The boomerang's distinctive sound and return flight pattern further analysed to understand controllable motion, rotation, angular shape with asymmetrical curves and the complex nature of aerodynamics: asymmetrical lift (Masi et al., 2002, p. 11).

- Maori Aotearoa-Maori astronomy impacted on many aspects of Maori culture, traditions and belief, from the origins of the Universe, to traditional calendrical systems, to the use of astronomy in language, architecture, agriculture and oceanic navigation (P. Harris et al., 2013, p. 325).

- First Nations Doig River Canada-The use of flaked rock carbon dated to $10,500 \mathrm{BC}$ knowledge was used to shape spear tips for the purpose of hunting and food gathering activities (Fladmark et al., 1988, p. 24).

While Indigenous people of the world possess an immense knowledge of their environments based on centuries of living close to nature, they struggle for recognition of their legitimate knowledge paradigms, with preferred philosophical beginnings (Henare, 1998). Today, the Indigenous physical artefact is available using an array of technology formats denoted as being the Indigenous IT artefact, an unreal digital version of the physically real artefact (Shedlock \& Vos, 2019).

The availability of the Indigenous IT artefact is creating considerable pressure for Indigenous communities including rising claims of appropriation of Indigenous knowledge Blackstock (2016), data sovereignty for Indigenous peoples Hazel et al. (2018) and colonizing impacts of technology Shedlock and Vos (2018) being reported by Indigenous researchers globally. To add to the complexity, debate is beginning to emerge between the digital and the natural human artefact Black (2014) and the resourcing required to house Indigenous intelligent agents (Shedlock \& Vos, 2019). The impacts on traditional practices, data protection and language retention to mention a few are immense.

\section{VR presence}

VR offers tremendous opportunities in the depiction of artefacts and environments that are otherwise impossible within the real-world (Chen et al., 2011). According to Riva et al. (2007), it is possible to describe VR in terms of human experience, using the concept of presence. VR is the medium able to induce the experience of presence in a computergenerated world usually defined as the sense of being there or the feeling of being located in a world that exists outside the self (Riva et al., 2007). As discussed by Fisher (1999), presence involves simulated interaction within an environment where the user is able to access multiple images or three-dimensional sound cues that give distance and direction information for proximate objects and events.

For Indigenous research, the VR setting is enabled through a perceived sense of place. Therefore, this setting must be embedded in real life situations, in temporally and spatially specific ways (Crang \& Thrift, 2000). A sense of place in virtual environments and real experiences is not just a consequence of being surrounded by a spatial setting but of being engaged in another place. A place is particular, unique, dynamic, and memorably related to other places, peoples, and events, and is therefore hermeneutic (Cameron $\&$ Kenderdine, 2007). Non-place introduces the proposition that non-places can be characterized as unthinking spaces, for example, spaces that provide little assistance to the thought processes of their occupants. Non places are spaces where thought thrives on transitions, thresholds and boundary conditions between the strange and the familiar (Coyne, 2007).

The notion of Indigenous identity is reflected in the study of Muriwai et al. (2015) who believes, those who are highly connected to their culture may experience better psychological outcomes. Therefore, presenting the location of one's virtualself in a VR setting is dependent on one's attention shifting from the physical environment to the VR environment. Any VR experience may require a succinct in-depth level of connection for Indigenous viewers using models, methods, objects and instantiations (Shedlock \& Vos, 2018).

Sense of self presence suggests three levels of self-identification corresponding to three distinct levels of presence (Riva et al., 2004). Further introduced as a preconscious forerunner of self with characterized notions of selfhood based on the following (Damasio, 2010):

- The proto-self-a coherent collection of neural patterns that map, moment by moment, the physical state of the organism. Differentiating the self from the external world through action. It depends on the level of awareness towards the surrounding environment that effects the movement or perception-action coupling self versus non-self.

- The core self - a transient entity that is continuously generated through encounters with objects. Successfully acting in the external world towards a present object. It depends on the level of connection clarity or vividness self versus present external world. 
- The extended self - a systematic record of the more invariant properties that the organism has discovered about itself. Successfully acting in the external world towards a possible object. It depends on the level of appropriateness self versus possible external world.

Further portrayed as a sense of embodied connection and, building on the work of Riva et al. (2004) such as showing a mental state of awareness with a coherent collection of logical neural patterns that map, moment by moment interactions. In this way, the Indigenous IT artefact delivers an embodied spatial connection to VR that involves memories, is spatially specific, is situated in time and, includes a place location (Cameron \& Kenderdine, 2007). As opposed to a monolithic external connection to objects of the unknown (von Thater-Braan, 2007).

However, finding a suitable method to model Indigenous data structures during the construction of the Indigenous IT artefact may add a further layer of complexity, that is, addressing vague Indigenous views towards the world as opposed to established binary encoded computing structures.

\section{Evolutionary fuzzy framed data structures}

One such coding method is in the field of Fuzzy Logic. Fuzzy logic systems (FLSs) have continued to attract interest working with linguistic descriptions to provide an efficient way to describe complicated terms using a set of organized process steps (Choi et al., 2015). The term fuzzy logic was introduced by Zadeh (1965) to model logical reasoning when dealing with vague or imprecise statements (Pelletier, 2000) such as Tama is tall or Tama is very tall. In this way, approximate reasoning (also called fuzzy reasoning) becomes a qualitative rather than quantitative measure (Zadeh et al., 1996). A method to organize language into formats using computer logic (Figallo \& Pelaitay, 2014) for example, "height" as a linguistic variable with values "short, tall, very short, very tall, not very short and not very tall."

FLS handle the concept of partial truth, where the truth value may range between completely true and completely false (Novak et al., 1999). Language rules form the computers logic where the truth can be established between values of zero (0) and one (1) for example 0.75 percent of truth. FLS uses three basic operations as probability sets of information being union, intersection and, complement. Subject rules are either provided by subject experts or are extracted from numbered sets of data using IF-THEN statements for example: IF Tama is " $A$, tall" and " $B$, quick," THEN Tama is suited to playing basketball. Ongoing, while input variables in mathematics usually take numerical values, in fuzzy logic applications, non-numeric values are often used to facilitate the delivery of each rule such as modifying each linguistic value with adjectives or adverbs (Huynh et al., 2002). The process of FLS consists of (1) inputs, fuzzification; (2) an inference engine containing a set of rules and (3) membership functions; (4) de-fuzzification; and (5) outputs (Zhuang \& Wu, 2001).
The fuzzy logic controller processes the inputs of information received through the rules, applying the language operator rules to infer the fuzzy actions (Gomes et al., 2010). Association rule based mining of a fuzzy system uses the rule to identify the correlations between levels of truth (Agrawal et al., 1993). The fuzzy rule is computationally stored and then processed, which is usually presented in terms of IF a language transaction is true THEN an understood rule applies. Using the same example from above: IF Tama is $A$, tall and $B$, quick, THEN Tama is suited to playing basketball.

The algorithm inference engine encodes the knowledge learned from if-then fuzzy rules, processing data using fuzzy principles to interpret and report the result (Bandyopadhyay \& Maji, 2012; Figueiredo \& Gomide, 1999). The fuzzy logic algorithm is an inference engine that behaves similar to that of the human brain, simulating human decision making by performing approximate reasoning to achieve a desired result strategy (Zhuang \& $\mathrm{Wu}, 2001)$. The final part of the process is defuzzification Cuong and Hai (2015) used to arrange the output into a "crisp" output value for reporting. In this way, the FLS houses a fuzzy logic controller (algorithm) where fuzzification entails the process of mapping mathematical input values into fuzzy membership functions (Ehsani et al., 2017).

\section{The IT VR artefact-Indigenous data filter}

The IT VR artefact is, presented as an Indigenous data filter (IDF) guided by a FLS. A system that applies a Typejuby Takagi-Sugeno model as well as the Tsukamoto output model during the controllers processing of its membership function. One where the FLS has degrees of membership between full crisp-member, (100\% membership) or partial member (between $0 \%$ and 100\% membership). Further defined as a membership function of a fuzzy set on the universe of discourse $\mathrm{X}$ where each element of $\mathrm{X}$ is mapped to a value between 0 and 1 . This value, called membership value or degree of membership, quantifies the grade of membership of the element in X to each fuzzy set.

The FLS applies two inputs as fuzzy sets with a single output. The IDF uses the process controller to explain a priori knowledge for Indigenous terms further defining the membership functions ability to perform sampling that involves communicating with the world of $\mathrm{X}$ that it knows about and, interacting with that worlds known threshold range in search of agreement. In this way, the IDF is a term used to describe Indigenous data sub-sets within a full data set for the purpose of benefitting Indigenous viewing, analysis and reporting. As an example, the IDF describes, how a computational model can be Indigenously organized, what Indigenous concepts and ideas can be implemented, where to find Indigenous data and, when evaluation may occur, at either the logic design or after the VR artefact has been constructed. One that involves an Indigenous level of understanding that connects Indigenous ideas and concepts as experts to form objects, models, methods and instantiations using technology. 


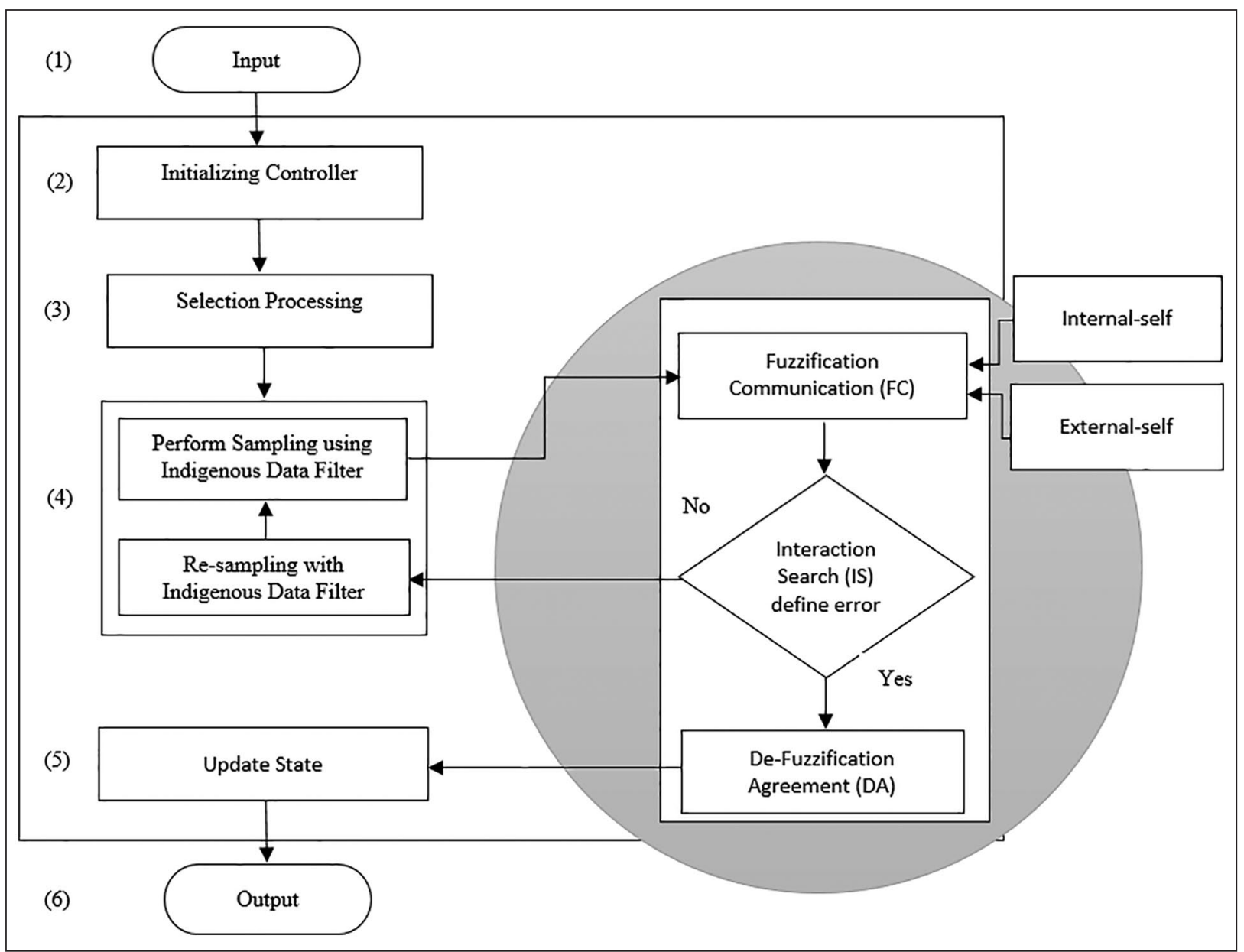

Figure I. Indigenous data filter.

The IDF is further described as a staged processing controller involving (1) data input (2) initializing the controller, (3) input selection processing, (4) performance sampling, (5) update state and, (6) data outputs. Figure 1 below demonstrates Indigenously modelled concepts for experiencing the VR artefact and connecting Indigenous environments $(\mathrm{X})$ to technology.

1. Data Input-Participants users enter the VR application and are directed through two scene's A then $\mathrm{B}$, and asked to respond to two questions as a format of self-assessment shown in Figure 2 below.

Input $\mathrm{A}-$ to enact external virtual-self, choose five images from the images provided.

Input B - to enact internal virtual-self, respond to the five questions provided.

The first question responds to the external virtual-self input and, asks the participant to 'Select $5 x$ images of interest to the user'? Ten images are randomly made available to the user interface, from a selection pool of 30 images. Indigenous images are placed in the array position $(0.0,0.2,0.4,1.1,1.3)$ shown above in Figure 2. Each image selection within the array is recorded with a weighted value
0.25 . This determines the participant's level of attention awareness towards their external virtual-self by locating Indigenous image concepts inside the VR setting.

The second grouped question responds to the internal virtual-self input using the silhouette image provided.

1. Respond to Q1: Locate your ears-Kei hea tō ringa (Where are your ears)?

2. Respond to Q2: Locate your heart-Kei hea tō manawa (Where is your heart)?

3. Respond to Q3: Locate your eyes - Kei hea tō karu (Where are your eyes)?

4. Respond to Q4: Locate your legs-Kei hea tō waewae (Where are your legs)?

5. Respond to Q5: Locate your stomach-Kei hea tō puku (Where is your stomach)?

Once the participant has successfully navigated via the two UI scenes, the participant then, teleports to the feature VR scene while the data inputs A and B is recorded and forwards to the controllers initialization stage for processing.

2. Initializing the controller-The main purpose for initializing the controller is to organize the membership function's condition by identifying initial value inputs in search of outputs. As shown in 


\begin{tabular}{|c|c|c|c|c|}
\hline \multicolumn{5}{|c|}{ (A) External } \\
Virtual-Self
\end{tabular}

Figure 2. Input Layer.

\begin{tabular}{|c|c|c|c|c|c|c|c|c|}
\hline \multirow{2}{*}{\multicolumn{5}{|c|}{$\begin{array}{c}\text { (A) External } \\
\text { Virtual-Self } \\
\text { Choose one }\end{array}$}} & $\begin{array}{l}\text { (B) Internal } \\
\text { Virtual-Self }\end{array}$ & \multicolumn{3}{|c|}{$\begin{array}{l}\text { (C) Indigenous } \\
\text { Virtual-Self }\end{array}$} \\
\hline & & & & & Select a physical location & External & Internal & Attention \\
\hline $\begin{array}{c}0.0 \\
\text { Img1 }\end{array}$ & $\begin{array}{c}0.1 \\
\text { Img2 }\end{array}$ & $\begin{array}{c}0.2 \\
\text { Img3 }\end{array}$ & $\begin{array}{c}0.3 \\
\text { Img4 }\end{array}$ & $\begin{array}{c}0.4 \\
\text { Img5 }\end{array}$ & & & & \\
\hline $\begin{array}{c}1.0 \\
\operatorname{Img} 6\end{array}$ & $\begin{array}{c}1.1 \\
\operatorname{Img} 7\end{array}$ & $\begin{array}{c}1.2 \\
\text { Img8 }\end{array}$ & $\begin{array}{c}1.3 \\
\text { Img9 }\end{array}$ & $\begin{array}{c}1.4 \\
\operatorname{Img} 10\end{array}$ & & & & \\
\hline
\end{tabular}

Figure 3. Initializing layer.

Figure 3 below, initialization is now aware of two unique input identifiers as linguistic terms where, $\mathrm{A}=$ External Virtual-Self and, B = Internal Virtual-Self, in detecting a level of $\mathrm{C}=$ Indigenous Virtual-Self connection.

Using a FLS Takagi-Sugeno model of theory, the controller applies input identifiers to A and B with the third identifier $\mathrm{C}$ being the output result. The controller is now ready for initialization using the Takagi-Sugeno fuzzy rule where: IF $x$ is A AND $y$ is B THEN $z$ is $f(x, y)$ where $\mathrm{x}, \mathrm{y}$ and $\mathrm{z}$ are linguistic variables while, the universe of discourse of A and B are fuzzy sets of a mathematical function (Takagi \& Sugeno, 1985).

$$
\text { if input } A=x \text { and input } B=y \text {, then outputC is } z=A x+B y
$$

Initializing the process controller is now one where $A(x)$ and $B(y)$ are the antecedent fuzzy sets and $z=f(x, y)$ is the consequent output function. Where $f(x, y)$ is a polynomial of the input membership function variables $(x)$ and $(y)$, able to function as both can appropriately describe the output of the model within the IDF system specified by the antecedent of the Type 2 Takagi-Sugeno rule.

Revisiting the research question: can VR image-processing techniques be modelled to connect Indigenous participant users to their digital selves? The two input variables link $(x, y)$ where the phrase participant users is the antecedent logic $(x)$ while their becomes the antecedent logic for $(y)$. To complete the function $(z)$ is the output channel representing the consequent logic result: $z=f(x, y)$.

3. Selection Processing-The two input variables now represent the external virtual-self $(x)$ and, the internal virtual-self $(y)$ and are now conditioned for selection processing. The third identifier represents the Indigenous virtual-self $(z)$ output variable. In this way, the IDF becomes an 


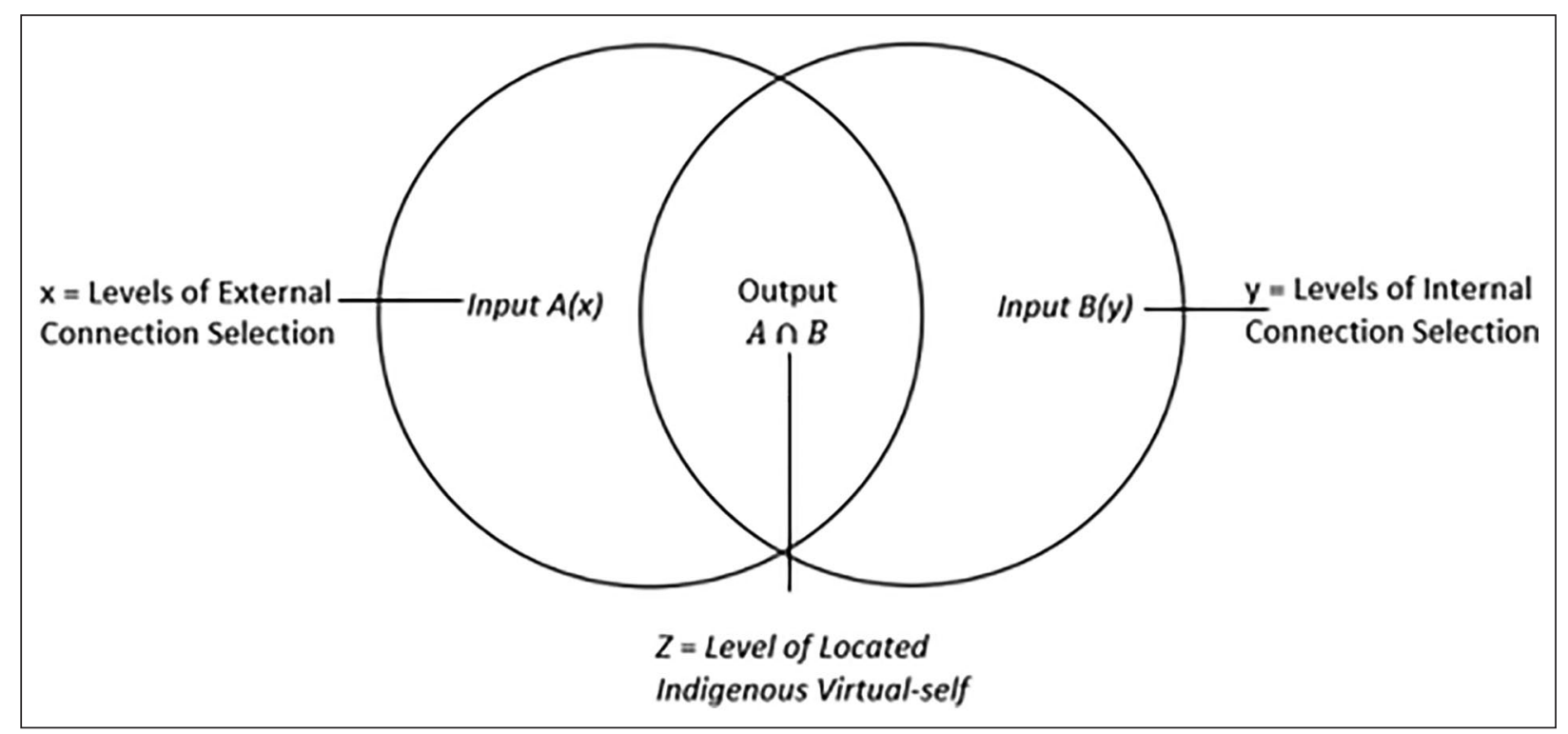

Figure 4. Sets for selection processing.

Indigenous problem solving tool using logic where: if $x$ is Aand $y$ is Bthen $z=f(x, y)$ shown in Figure 4 below as output $A \cap B$.

The fuzzifier selection processor prepares the data set for further sampling. Linguistic terms also known as fuzzy hedges are formulated as fuzzy rule sets to arrange membership functions. The formulated rules relate the inputs to outputs using IF-THEN statements. The number of membership functions needed to explain combinations for the two input variables and one output variable is $5 \times 5$ total 25, outlined in Table 1.

As described in Table 1, there are five inputs shown in the external — self $(x)$ column labelled, NOT LIKELY, LESS LIKELY, LIKELY, MORE LIKELY, VERY LIKELY. In addition, there are five inputs in the internal-self $[y]$ column labelled VERY LOW, LOW, AVERAGE, HIGH, and VERY HIGH. The virtual-self column is linked to the level of user connectedness output. This is shown as being LOW, MODERATE, AVERAGE, SIGNIFICANT, and HIGH. As an example: IF Indigenous external-self is more-likely (0.75) and Indigenous internal-self is very-high (1), the weighted average of both external and internal self is calculated as a high (0.75), level of virtual-self connection.

4. Performance Sampling — The controller's performance sample processing is ready for evaluation using IF-THEN membership rules to achieve a priori measure. In this way, the rule is a numerical data set expressed as a collection of IF-THEN statements available for sampling. As in classical logic, the FLS adopts the union operations on fuzzy sets advancing the IF-THEN rule-based system. The IF part of the rule termed $x$ is $A$ the antecedent with $y$ is $B$ the antecedent forming subsets to the premise. The THEN part of the rule $z$ is $C$ the consequent or output result of the membership rule.
Let $\mu_{\mathrm{A}}$ and $\mu_{\mathrm{B}}$ be a union of membership functions that define the fuzzy sets $A$ and $B$, respectively, on the universe $X$. To evaluate the conjunction of the rule antecedent, we apply the AND operation as the intersection of fuzzy sets to A and B members. Operators become the subjects and verbs of logic expressed as rules such as the format: If $x$ is $\mathrm{A}$ and $y$ is B THEN record output $\mathrm{z}$. As an example, IF antecedent THEN consequent written using logic: IF $x$ is $A$, AND, $y$ is $B$ the weighted aggregate of both result in an output value $z$. where: $x \cap y=z$

$$
w_{i}=\operatorname{AND}\left(F_{1}(x),\left(F_{2}(y)\right)\right.
$$

This step is calculated using the relevance to the antecedents, combined with the methods of connection (AND) for the extraction of a single value. From this position, the resulting value is ready to generate fuzzy membership functions as the MIN weighted average input-output dataset using the format: if $x$ is A and $y$ is $B$ then $z=f(x, y)$. Figure 5 below shows the firing calculation for sets $A$ AND B where $W_{1,2}$ become the membership functions for input $W_{1}$ AND $W_{2}$ adopting the Tsukamoto output model.

Statements in the multiple conjunctive antecedent (not consequent) part of the rule involve fuzzy logical connectives AND. The standard logical operations x AND $\mathrm{y}$ is used to determine the output using the weighted average function $z=\frac{w_{1}, z_{1}+w_{2,} z_{2}}{w_{1}+w_{2}}$

The IDF is ready to fire with any output functions that do not meet any filtering thresholds returning to the filtering system for re-sampling while outputs that do meet the filtering thresholds progressing to the update state.

5. Update State-The IDF filtering process is applied to update the state before proceeding to the data output phase of the IDF. Thresh-hold conditions have been satisfied and data outputs forwarded for reporting. 
Table I. Membership rules.

\begin{tabular}{|c|c|c|c|c|c|c|}
\hline Rule & Internal-self (input) & $x$ & External-self (input) & $y$ & Virtual-self (Output) & $z$ \\
\hline I & Not-likely & 0 & Very-low & 0 & LOW & -1.0 \\
\hline 2 & Not-likely & 0 & Low & 2.5 & Moderate & -0.75 \\
\hline 3 & Not-likely & 0 & Average & 5 & Average & -0.50 \\
\hline 4 & Not-likely & 0 & High & 7.5 & Significant & -0.25 \\
\hline 5 & Not-likely & 0 & Very-high & 1 & $\mathrm{HIGH}$ & 0 \\
\hline 6 & Less-likely & 2.5 & Very-low & 0 & LOW & -0.75 \\
\hline 7 & Less-likely & 2.5 & Low & 2.5 & Moderate & -0.5 \\
\hline 8 & Less-likely & 2.5 & Average & 5 & Average & -0.25 \\
\hline 9 & Less-likely & 2.5 & High & 7.5 & Significant & 0 \\
\hline 10 & Less-likely & 2.5 & Very-high & I & $\mathrm{HIGH}$ & 0.25 \\
\hline II & Likely & 5 & Very-low & 0 & LOW & -0.5 \\
\hline 12 & Likely & 5 & Low & 2.5 & Moderate & -0.25 \\
\hline 13 & Likely & 5 & Average & 5 & Average & 0 \\
\hline 14 & Likely & 5 & High & 7.5 & Significant & 0.25 \\
\hline 15 & Likely & 5 & Very-high & I & $\mathrm{HIGH}$ & 0.5 \\
\hline 16 & More-likely & 7.5 & Very-low & 0 & LOW & -0.25 \\
\hline 17 & More-likely & 7.5 & Low & 2.5 & Moderate & 0 \\
\hline 18 & More-likely & 7.5 & Average & 5 & Average & 0.25 \\
\hline 19 & More-likely & 7.5 & High & 7.5 & Significant & 0.5 \\
\hline 20 & More-likely & 7.5 & Very-high & I & $\mathrm{HIGH}$ & 0.75 \\
\hline 21 & Very-likely & 1 & Very-low & 0 & LOW & 0 \\
\hline 22 & Very-likely & 1 & Low & 2.5 & Moderate & 0.25 \\
\hline 23 & Very-likely & 1 & Average & 5 & Average & 0.5 \\
\hline 24 & Very-likely & I & High & 7.5 & Significant & 0.75 \\
\hline 25 & Very-likely & 1 & Very-high & I & $\mathrm{HIGH}$ & I \\
\hline
\end{tabular}

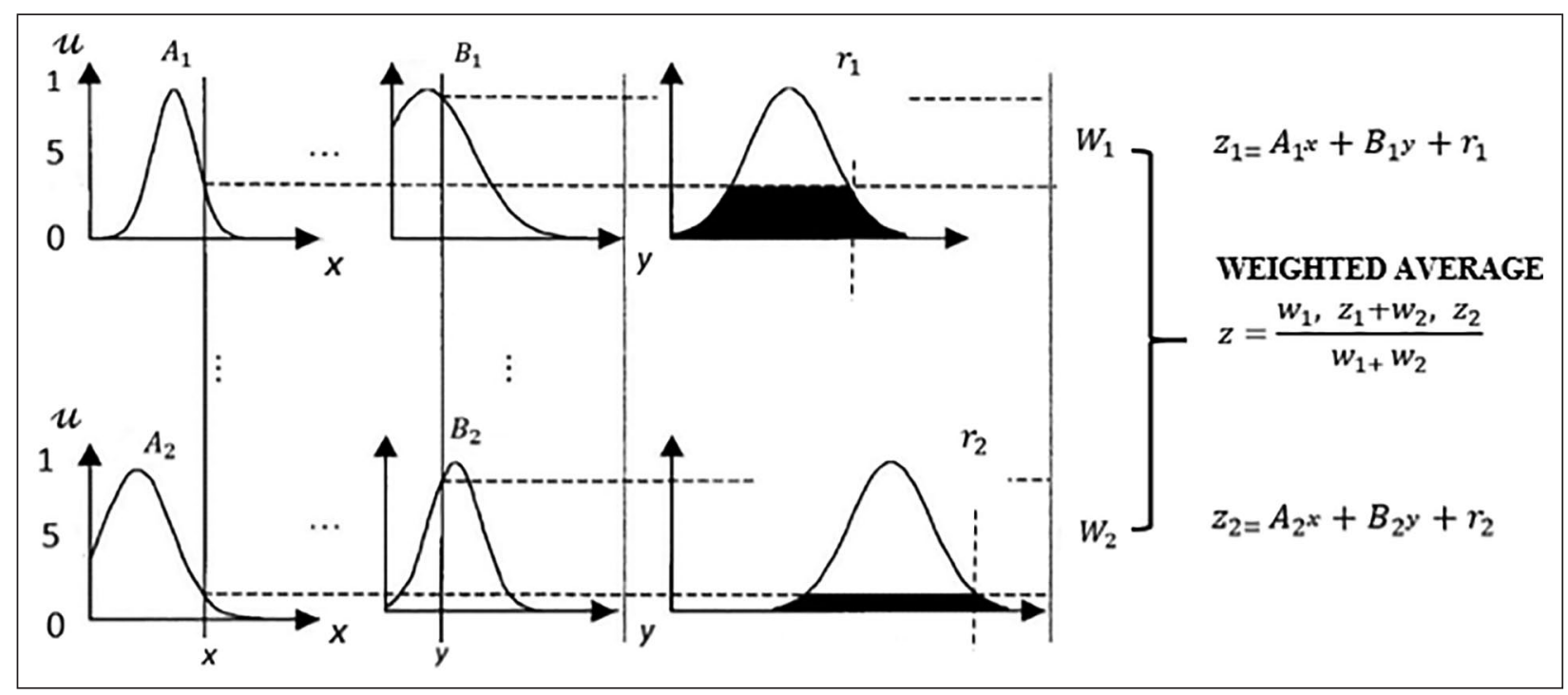

Figure 5. Perform sampling.

6. Data Outputs - The data output is reported outlining the levels of Indigenous connection to the VR setting on entry to the VR scene.

The Indigenous data filtering process now reveals both computation and graphical reports as discretely organized data sets and graphic displays using tactics such as sigmoid curve, quadratic and cubic polynomial curve displays. Furthermore, sampling uses nonlinear terms within a local linear controller to define the role of the membership function to better-understand a level of connectedness of the Indigenous virtual-self inside a VR setting.

\section{Evaluation}

Evaluation involves the IT artefact compared to the literature and then, reported as a research activity. This study is based on the research question: can VR image-processing techniques be modelled to connect Indigenous participant users to their digital selves? To respond to the question, 
three avenues are pursued as an approach. An Indigenous post-colonial research paradigm to provide an over-arching focus during the construction of the IT artefact. VR is employed as the technology medium and, Fuzzy Logic is used as a functional method linking Indigenous concepts and ideas to the VR technology.

In regards to Indigenous research, the literature shows Indigenous research activities producing artefacts as a science have been in existence over hundreds of thousands of years. Indigenous science tended to be enacted "living in tandem with nature" uniquely aware of the environment and spatially connected, a result of long-term occupancy and a state of being situated to that space both lived and in memory. The IT artefact seeks to adopt an Indigenous context further defined as one that pursues Indigenous priorities using concepts and ideas characterized as property types to communicate Indigenous objects, models, terminologies and languages alike. Ongoing, postcolonial Indigenous research operating in the space of technology is becoming an emerging topic open for further investigation, however, limited material in the format of publication or journals exist for digital technology research using an Indigenous post-colonial approach opening future research avenues.

For VR, the literature views the basic function of VR presence as a way of being inside a computer aided experience that allows the virtual self to experience located differences between the real world and digital world and to identify any distinctions between either. An experience that includes a characterized reflection of both an external mode and internal mode of dependency connection to obtain an understood level of connection to any Indigenous virtual self. As a result, the IT artefact is modelled as a self-assessment tool in search of an Indigenous VR connection using known real world objects, models, terminologies and languages. One that has two inputs as a set of VR questions ex-ante to determine the level of connection to the participant users internal self and external self. Furthermore, processing shows an IDF modelled IT artefact focused on Indigenous participant users as a technical tool of measurement within a VR computer aided three-dimensional system.

FLS theory provides an avenue for Indigenous research to align with probability logic. This opens ongoing opportunities to create Indigenously framed discrete models and methods using vague reporting systems. Ongoing, discrete modelling provides an ideal scenario to examine and compare Indigenous data sets in search of an Indigenous virtual-self output. The study reveals a design model and algorithm structure to address a key tension for Indigenous VR in research, ascertaining a level of Indigenous VR connection using an internal and external VR sensory connection.

Now, a modelled process to Indigenously communicate and interact when reaching agreement provides a strong platform for future ongoing work using an IDF. More importantly, agreement is formed using Indigenous relationships rather than touting position within a eurocentric model of research. This is a critical difference between both Indigenous and eurocentric research when discussing agreement in a technological setting. One where agreement is computationally based on Indigenous relationships as opposed to touting position towards optimization.

In summary, the IT artefact describes how a computational model can be Indigenously organized, what Indigenous concepts and ideas can be implemented, where to find Indigenous data and, when evaluation may occur, at either the logic design or after the VR has been constructed. One that involves an Indigenous level of understanding that connects Indigenous ideas and concepts as experts to form objects, models, methods and instantiations using technology. In this way, the IDF is a model used to focus on Indigenous data sub-sets within a full data set that benefits Indigenous participant users within a VR setting.

Three notable challenges are exposed moving forward for any IDF, (a) the expertise required to understand Indigenous input systems when processing Indigenous data, which was not investigated with any vigour during the study and (b) the processing of Indigenous data requirements becoming a lot more complex making any IDF both time constrained and resource intensive. Third, Indigenous research in the space of VR and logical data modelling is scarce. Indigenous researchers must continue to challenge technology concepts emanating from individual knowledge, moving towards one that is relationally bound as a shared collective, and continuing to progress the work of earlier Indigenous researchers.

\section{Discussion}

Today, the Indigenous physical artefact is available using an array of technology formats such as VR denoted as being the IT artefact, an unreal digital version of the physical real artefact. Postcolonial Indigenous research operating in the space of technology is a topic open for further investigation. Indigenous researchers must continue to pursue technology concepts during the construction of any IT artefact, moving towards becoming relationally bound as a shared collective in search of agreement as opposed to agreement that touts position in the search of optimization. This is achievable through the construction of Indigenous technological models, frameworks and systems when researching science activities.

The IDF model involves an Indigenous level of understanding that connects Indigenous ideas and concepts to form objects, models, methods and instantiations using technology. The model describes how a computational theory model can be Indigenously organized, what Indigenous concepts and ideas can be implemented, where to find Indigenous data and, when evaluation may occur, at either the logic design or after the VR has been constructed. For this investigation, evaluation was an ex-ante activity opening future opportunities to test the model post construction of the VR artefact. To keep the study confined, only a small portion of Indigenous literature was used to introduce Indigenous paradigms, sciences and technology leaving open future research to advance an Indigenous technology cause. Given the massive amount of Indigenous knowledge concepts available to technology, ongoing 
research to align with technical data structures was left open for new research activities to occur.

For VR - this research reveals a theoretically modelled VR setting that portrays a level of Indigenous virtual-self, operating inside the VR artefact. As a result, three forms of Indigenous VR are presented to digitally locate themselves using a format of self-assessment. (1) the Indigenous virtual internal-self and how the user locates their thinking self, based on their understanding of Indigenous language, and objects, (2) the Indigenous virtual external-self and how the user interacts with Indigenous language and objects and, (3) the Indigenous virtual-self calculated using both results from internal self and external self-as a computational fuzzy system.

Finally, FLS theory provides an avenue for Indigenous research to align with probability logic in the context of an Indigenously constructed VR setting. This opens opportunities to create discrete models and methods using vague systems. Furthermore, discrete modelling provides an ideal scenario to pursue quantitative and, qualitative analysis between Indigenous internal/ external-self inputs in search of the Indigenous virtual-self.

\section{Conclusion}

The research question addressed by this research is; can VR image-processing techniques be modelled to connect Indigenous participant users to their digital selves? The investigation shows a way to organize a modelled IDF system that can be employed to connect Indigenous users to their digital virtual selves. The model describes how a computational model can be Indigenously organized, what levels of expert understanding is required to implement Indigenous concepts and ideas and, where to find Indigenous data and, when evaluation may occur, at either the logic design or after the VR has been constructed.

Contributions are made to the body of Indigenous research and, Indigenous participant users of VR. Indigenous research is provided with a modelled VR artefact that describes a version of the digital Indigenous virtual-self demonstrating a broad group of Indigenous dependencies linked to the internal virtual-self and external virtual-self. Now, Indigenous participant users of VR may access a digitally modelled self-assessment tool to identify a level of their Indigenous digital self.

Three notable research windows open for the future. First, the IDF is modelled to receive inputs from participant user's ex-ante, opening study for the IDF to impact or coheres user feedback after the VR experience ex-post. Second, the filter processing requirements of the IDF may become more time constrained and resource intensive, further research could address these resource constraints such as fine-tuning the FLS membership function. Finally, limited publications exists matching indigeneity to technology in a modern era. Indigenous researchers must continue to research technology concepts for themselves moving beyond a eurocentric approach emanating from individual knowledge, to one that is relationally bound as a shared collective. An Indigenous technology paradigm that involves Indigenous technology models, Indigenous frameworks and Indigenous systems is required.

\section{Limitations}

Indigenous methodology for discrete data structures requires ongoing investigation. Furthermore, this paper represents a theoretical approach presented as a proof of concept. No discrete testing was applied to the model, opening future work and the construction of the VR artefact as a further exemplar of Indigenous work in practice.

\section{Declaration of conflicting interests}

The author(s) declared no potential conflicts of interest with respect to the research, authorship, and/or publication of this article.

\section{Funding}

The author(s) received no financial support for the research, authorship, and/or publication of this article.

\section{ORCID iD}

Kevin Shedlock (DD https://orcid.org/0000-0001-6032-1744

\section{Glossary}

Kei hea tō ringa?Where are your ears? Kei hea tō manawa?Where is your heart?

Kei hea tō karu? Where are your eyes?

Kei hea tō waewae? Where are your legs? Kei hea tō puku?Where is your stomach?

\section{References}

Agrawal, A. (1995). Indigenous and scientific knowledge: Some critical comments. http://journal.ui.ac.id/index.php/jai/article /view/3331/2618

Agrawal, R., Imielinski, T., \& Swami, A. (1993). Mining association rules between sets of items in large databases. International Conference on Management of Data, 22(2), 207-216.

Bandyopadhyay, S., \& Maji, B. (2012). Antenna array synthesis using Tsukamoto fuzzy logic controller. National Conference on Emerging Trends in Electronics \& Telecommunication Engineering. https://ieeexplore.ieee.org/document/6659353

Battiste, M., Bell, L., \& Findlay, L. (2002). Decolonizing education in Canadian universities: An interdisciplinary, international, Indigenous research project. Canadian Journal of Native Education, 26(2), 82-95.

Bennett, E. (2012). Indigenous methodologies: Characteristics, conversations, and contexts by Margaret Kovach. The Canadian Geographer / Le Géographe Canadien, 56(2), 292-293. https://doi.org/10.1111/j.1541-0064.2012.00420.x

Bird, S. E. (2018). Dressing in feathers the construction of the Indian in American popular culture. Routledge. http://public. eblib.com/choice/PublicFullRecord.aspx?p=5294887

Black, D. (2014). Where bodies end and artefacts begin: Tools, machines and interfaces. Body \& Society, 20(1), 31-60. https://doi.org/10.1177/1357034X13506946

Blackstock, C. (2016). The breath of life versus the embodiment of life: Indigenous knowledge and western research. https:// www.researchgate.net/publication/237555666_The_breath_ 
of life versus the embodiment of life Indigenous knowledge_and_western_research

Boxall, P. C., Englin, J., \& Adamowicz, W. L. (2003). Valuing aboriginal artifacts: A combined revealed-stated preference approach. Journal of Environmental Economics and Management, 45(2), 213-230. https://doi.org/10.1016/S0095 -0696(02)00063-3

Cameron, F., \& Kenderdine, S. (Eds.). (2007). Theorizing digital cultural heritage: A critical discourse. MIT Press.

Chen, J. F., Warden, C. A., Wen-Shung Tai, D., Chen, F.-S., \& Chao, C.-Y. (2011). Level of abstraction and feelings of presence in virtual space: Business English negotiation in Open Wonderland. Computers \& Education, 57(3), 21262134. https://doi.org/10.1016/j.compedu.2011.05.017

Chilisa, B. (2012). Indigenous research methodologies. SAGE.

Choi, H. D., Ahn, C. K., Shi, P., Lim, M. T., \& Song, M. K. (2015). L2-L $\infty$ Filtering for Takagi-Sugeno fuzzy neural networks based on Wirtinger-type inequalities. Neurocomputing, 153, 117-125. https://doi.org/10.1016/j.neucom.2014.11.046

Coyne, R. (2007). Thinking through virtual reality: Place, nonplace and situated cognition. Virginia Tech, 10. https:// scholar.lib.vt.edu/ejournals/SPT/v10n3/coyne.html

Crang, M., \& Thrift, N. J. (Eds.). (2000). Thinking space. Routledge.

Cuong, B. C., \& Hai, P. V. (2015). Some Fuzzy Logic operators for picture fuzzy sets (pp. 132-137). https://doi.org/10.1109/ KSE.2015.20

Damasio, A. (2010). Self comes to mind: Constructing the conscious brain. William Heinemann.

Dei, G. J. S. (2000). Rethinking the role of Indigenous knowledges in the academy. International Journal of Inclusive Education, 4(2), 111-132. https://doi.org/10.1080/136031100284849

Ehsani, E., Kazemi, N., Olugu, E. U., Grosse, E. H., \& Schwindl, K. (2017). Applying fuzzy multi-objective linear programming to a project management decision with nonlinear fuzzy membership functions. Neural Computing and Applications, 28(8), 2193-2206. https://doi.org/10.1007/s00521-0152160-0

Figallo, A. V., \& Pelaitay, G. (2014). Tense operators on De Morgan algebras. Logic Journal of IGPL, 22(2), 255-267. https://doi.org/10.1093/jigpal/jzt024

Figueiredo, M., \& Gomide, F. (1999). Design of fuzzy systems using neurofuzzy networks. IEEE Transactions on Neural Networks, 10(4), 815-827.https://doi.org/10.1109/72.774229

Fisher, S. (1999). Virtual environments, personal simulation \& telepresence. http://citeseerx.ist.psu.edu/viewdoc/ download?doi $=10.1 \cdot 1 \cdot 467.7055$

Fladmark, K., Driver, J., \& Alexander, D. (1988). The Paleoindian component at Charlie Lake Cave, British Columbia. The Society for American Archaeology.

Freire, P. (2000). Pedagogy of freedom: Ethics, democracy, and civic courage. Rowman \& Littlefield.

Gomes, R., Machado, L., \& De Moraes, R. (2010). A fuzzy logic based assessment tool for VR simulated medical environments. CDROM.

Grant, S., Dyson, L. E., \& Robertson, T. (2010). A participatory approach to the inclusion of Indigenous Australians in information technology. https://oi.org/10.1145/1900441 .1900480

Gregor, S., \& Hevner, A. (2013). Positioning and presenting design science research for maximum impact. MIS Quarterly, 37(2), $337-356$

Harris, A. (2014). Circulating cultures: Exchanges of Australian Indigenous music, dance and media. http://proxy.cm .umoncton.ca/login?url=http://www.jstor.org/stable/10 $.2307 / j . c t t 13 w w v 9 j$
Harris, P., Matamua, R., Smith, T., Kerr, H., \& Waaka, T. (2013). A review of Maori astronomy in Aotearoa-New Zealand. Journal of Astronomical History and Heritage, 16(3), 325-336.

Hartwell, R. M. (2017). The industrial revolution and economic growth. Methuen.

Hazel, J.-A., Martin, W.-J., Shep, S., Merrit, T., Meredith, P., \& Hall, M. (2018). Science for technological innovation (p. 14) [Māori Data Futures-Hui Report]. https://www. sftichallenge.govt.nz/

Henare, M. (1998). 'Te tangata, te taonga, te hau: Maori concepts of property. Property and the Constitution, Wellington for the Laws and Institutions.

Hennessy, K., \& Nathan, L. P. (2014). Honoring protocol: Design by, for and with aboriginal peoples. https://doi.org/10 $.1145 / 2598784.2611381$

Henry, E., \& Pene, H. (2001). Kaupapa Maori: Locating Indigenous ontology, epistemology and methodology in the academy. Organization, 8(2), 234-242. https://doi.org/10 $.1177 / 1350508401082009$

Hevner, A., \& Chatterjee, S. (2010). Design research in information systems (Vol. 22). Springer. http://link.springer. com/10.1007/978-1-4419-5653-8

Hsu, M., Howitt, R., \& Chi, C.-C. (2014). The idea of 'Country': Reframing post-disaster recovery in Indigenous Taiwan settings: The idea of "Country.." Asia Pacific Viewpoint, 55(3), 370-380. https://doi.org/10.1111/apv.12058

Huynh, V., Ho, T., \& Nakamori, Y. (2002). A parametric representation of linguistic hedges in Zadeh's fuzzy logic. International Journal of Approximate Reasoning, 30, 203-223.

Johnson, J. T., Howitt, R., Cajete, G., Berkes, F., Louis, R. P., \& Kliskey, A. (2016). Weaving Indigenous and sustainability sciences to diversify our methods. Sustainability Science, 11(1), 1-11. https://doi.org/10.1007/s11625-015-0349-x

Masi, S., De Bernardis, P., De Troia, G., Giacometti, M., Iacoangeli, A., Piacentini, F., Polenta, G., Ade, P. A. R., Mauskopf, P. D., Bock, J. J., Bond, J. R., Contaldi, C. R., Pogosyan, D., Prunet, S., Borrill, J., Boscaleri, A., Pascale, E., Coble, K., Farese, P., \& Romeo, G. (2002). The BOOMERanG experiment and the curvature of the universe. Progress in Particle and Nuclear Physics, 48(1), 243-261. https://doi.org/10.1016/S01466410(02)00131-X

Muriwai, E., Houkamo, C. A., \& Sibley, C. G. (2015). Culture as cure? The protective function of Māori cultural efficacy on psychological distress. New Zealand Journal of Psychology, 44(2), 14-24.

Novak, V., Perfilieva, I., \& Mockor, J. (1999). Mathematical principles of fuzzy logic. Kluwer Academic.

Pelletier, F. J. (2000). Meta-mathematics of fuzzy logic. Bulletin of Symbolic Logic, 6(3), 342-346. https://doi. org/10.2307/421060

Pidgeon, M. (2019). Moving between theory and practice within an Indigenous research paradigm. Qualitative Research, 19(4), 418-436. https://doi.org/10.1177/1468794118781380

Pries-Heje, J., Bakersville, R., \& Venable, J. (2008). Strategies for Design Science Research evaluation. In ECIS 2008 proceedings. https://aisel.aisnet.org/cgi/viewcontent.cgi?arti $\mathrm{cle}=1214 \&$ context $=$ ecis 2008

Riva, G., Mantovani, F., Capideville, C. S., Preziosa, A., Morganti, F., Villani, D., Gaggioli, A., Botella, C., \& Alcañiz, M. (2007). Affective interactions using virtual reality: The link between presence and emotions. Cyberpsychology \& Behavior, 10(1), 45-56. https://doi.org/10.1089/cpb.2006.9993

Riva, G., Waterworth, J., \& Waterworth, E. (2004). The layers of presence: A bio-cultural approach to understanding presence 
in natural and mediated environments. Cyberpsychology \& Behavior, 7(4), 402-416.

Shedlock, K., \& Vos, M. (2018). A conceptual model of Indigenous knowledge applied to the construction of the IT artefact. https://www.citrenz.ac.nz/conferences/2018/pdf /2018CITRENZ_1_Shedlock_Indigenous.pdf

Shedlock, K., \& Vos, M. (2019). Indigeneity and technology: Assigning Indigenous properties to the agent-based IT artefact ensemble. Pacific Asia Conference on Information Systems, Xi'an, China. https://aisel.aisnet.org/pacis2019/164/

Shedlock, K., Vos, M., \& Chard, S. (2016). Design Science Research: Building evaluation into the construction of Indigenous cultural artefacts in New Zealand. Australasian Conference on Information Systems, Wollongong, NSW, Australia. https://aisel.aisnet.org/acis2016/41/

Smith, L. T. (1999). Decolonizing methodologies: Research and Indigenous peoples. Zed Books; University of Otago Press.

Suh, S. C., Tanik, U. J., Carbone, J. N., \& Eroglu, A. (Eds.). (2014). Applied cyber-physical systems. Springer. https://doi. org/10.1007/978-1-4614-7336-7

Takagi, T., \& Sugeno, M. (1985). Fuzzy identification of systems and its applications to modeling and control. IEEE
Transactions on Systems, Man, and Cybernetics, 15(1), 116-132. https://doi.org/10.1109/TSMC.1985.6313399

Tuhiwai, L., \& Reid, P. (2000). Maori research development. Kaupapa Maori principles and practices. http://www. rangahau.co.nz/assets/SmithL/Maori_research.pdf

von Thater-Braan, R. (2007). Is it possible to have information technology that reflects Indigenous consciousness. Proceedings of the Native Science Academy. http://planetmaori.com/ Files/Content/2014/IT4IndigenousConsciousness.pdf

Wilson, S. (2001). What is Indigenous research methodology. Canadian Journal of Native Education, 25(2), 175-179.

Wilson, S. (2003). Progressing towards an Indigenous research paradigm in Canada and Australia. Canadian Journal of Native Education, 27(2), 161-178.

Zadeh, L. (1965). Fuzzy sets. Information and Control, 8(3), 338-353. https://doi.org/10.1016/S0019-9958(65)90241-X

Zadeh, L., Klir, G. J., \& Yuan, B. (1996). Fuzzy sets, fuzzy logic, and fuzzy systems: Selected papers. World Scientific.

Zhuang, H., \& Wu, X. (2001). Membership function modification of fuzzy logic controllers with histogram equalization. IEEE Transactions on Systems, Man and Cybernetics, Part B (Cybernetics), 31(1), 125-132. https://doi.org/10 $.1109 / 3477.907571$ 